\title{
Developmental aspects of three dimensional concept attainment'
}

\section{DAVID R. STONE, DEPARTMENT OF PSYCHOLOGY, UTAH STATE UNIVERSITY, Logan, Utah 84321}

Students (N-470) of grades $1-9$ were directed to place 54 blocks which varied in color, size, and shape into a three dimensional sorting bin, containing a like number of compartments. The $S$ had to simultaneously take into account three dimensions related to color, size, and form. The task required that logical structure would override "concrete" sensory ordering. While overly "concrete" responses to selected attributes extended strongly into the ninth grade, significant system differences began to appear at the sixth grade level. Discrimination training significantly increased the scores.

The question of how students achieve solutions to problems involving concept attainment is part of a growing field of interest in mediation and cognition. Piaget (Flavell, 1963, p. 194) described a two dimensional categorizing of objects varied in size and color. He characterized the cognitive "operation" required as "double seriation". This same process is, of course, widely used in the double-axis scattergram. Bruner (1964) introduced the variation of having students reverse a two dimensional sorting procedure which involved shifting a system of size and height variations from small-short to large-high, and back again. He used a preplaced object as an initial guiding cue, and found significant age differences in the transformation task.

The method of this study extends the categorizing task to three dimensions. In solving the problem, the hierarchic transformations required by formal structure must override (White, 1965) concrete or unsystematized solutions for the best possible score to be attained.

Hypotheses. The major hypotheses of this study were: (1) students at developmental levels from first through ninth grade will demonstrate an increasing ability to solve a three-dimensional classification problem, (2) a training on object discrimination will improve performance.

Method. The Ss consisted of school children of grades 1 through 9. There were $360 \mathrm{Ss}$ ( $20 \mathrm{M}$ and $2 \mathrm{~F}$ in each grade) who received instructions to: "Place the blocks in the small boxes in each tray using the best plan you can, so that you could remember where they are with your eyes closed." An additional $10 \mathrm{Ss}$ at each grade level received instructions relating to perceptual features of the blocks, and a special ninth grade group of 20 received additional training on the structure of the sorting boxes. This group was also given the DAT spatial sub-test.

The equipment used consisted of 54 blocks based on the six solid polyhedra shown in Fig. 1. There were three sizes of each form, and three colors (black, white, yellow) of each size.

The Ss, of course, did not have to know the names of the blocks in order to perform the task.

The Ss were to put the blocks into the sorting-bin matrix which consisted of three movable layers $3 \times 3=6$ in each layer, as shown in Fig. 2. Each tray had 18 small compartments, which could accommodate any of the blocks being used. The layers were close, so that the set could be perceived as a unit of $3 \times 3 \times 6$ dimensions. Two preplaced blocks made only the planned solution possible. However, intermediate solutions, based on putting similar blocks together, or a series of logical "clusters" allowed points to be scored at all levels.

A perfect solution, as regulated by the preplaced blocks, required positioning all small blocks in the top tray, medium sized in the middle, (based on a medium black block in the middle layer, front face) and large in the bottom (based on a large white figure on the bottom layer, back face). The black were thus all in the front face, the yellow in the middle, and white in the back tier. This required a logical reversal of the developmental

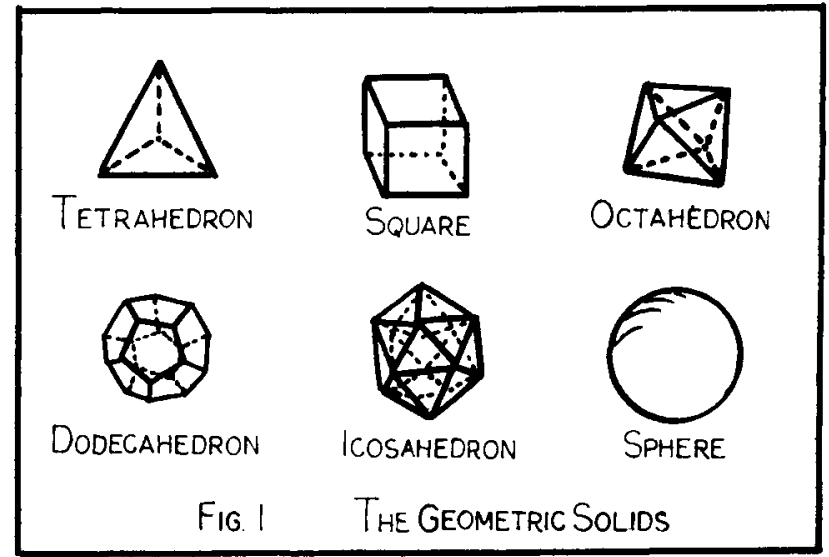

tendency, found in a pilot study, to put colors in "layers" rather than "slices."

In addition, since the forms could then be placed in the six box rows in order of increasing number of sides, it was possible to measure the changing accommodation for form progression, in addition to color and size. When students used identity grouping as a pairing, or used seriation for size, or recognized differences in the blocks, predetermined scoring points were given even if the solution was only a partial one. Inter-rater reliability of .98 was assured, because all possible identity and progression clusters were simply listed, with increasing points for each level of system complexity.

Results. Analysis of covariance (with the CTMM as the measurement of intelligence) was used in the computation of signifcant differences. An increasing ability to take three variables into account concurrently was observed. Significant system differences (5\% level) first appeared at the sixth grade as compared to Grades 1,2 , and 3. This supports Piaget's theory of cognitive development in that the results of the formal and reversible processes needed to achieve this solution were appearing at the ages of 11-12.

Significant differences (5\% level or better) appeared first for color, then for size and then for form progression when the performance of successive grades were analyzed. Early scoring patterns resulted from using two or three similar colors or large sizes together, a function of perception, rather than cognition.

While no absolute scoring is possible, a usable degree of difference was evidenced in terms of the significant differences which did develop, showing that the scoring system allowed

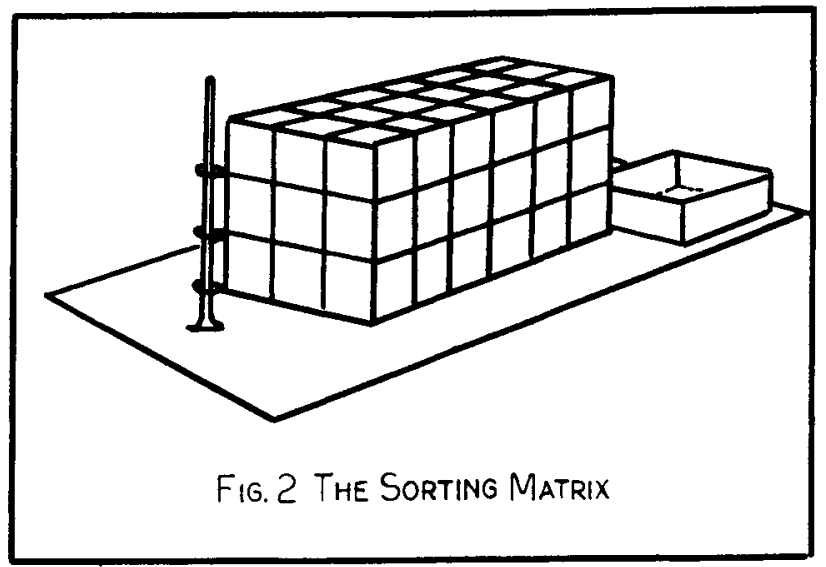


developmental (age) difference to be observed.

The results of discrimination training show highly significant (1\% level) improvement on the overall sorting. The procedure in al! othier aspects was the same as with the regular groups.

When spatial ability, as measured by a sub-test of the DAT, was correlated with the solutions of the ninth graders the $r$ was .11 for vertical scores and -.07 for horizontal. The solution was thus more related to maturation rather than to such spatial ability. Correlation with the nonlanguage section of the CTMM was.14, and for the language section, .04, again suggesting that differences were developmental.

Discussion. By assimilating or accommodating the preplaced blocks into a logically structured plan, a $\mathrm{S}$ using the best solution could attain the simultaneous placement of the six kinds, three colors, and three sizes into the $3 \times 3 \times 6$ matrix. Intermediate solutions could be scored in terms of identical pairs, trios, etc., and in terms of progressions of forms by increasing numbers of sides.

The increasingly formal and logical structuring shown in the score data may be interpreted as supporting a developmental theory of cognitive development along lines proposed by Piaget.
Students were required to "decenter" from focusing on a particular perceptible attribute, such as color, and to take all factors into consideration, systematically.

Training in discrimination has a strongly facilitating effect on overall performance for all groups studied, but no sex differences were found in any of the variables, or combinations of variables.

The Ss occasionally showed evidence of an "alternation" behavior in that having worked with one attribute they shifted to another, but without a unified mode of attack, especially at the early ages.

\section{REFERENCES}

BRUNER, J. S. The course of cognitive growth. Amer. Psychol., 1964, 19, 1, 1-15.

FLAVELL, J. H. The developmental psychology of Jean Piaget. Princeton, New Jersey: Van Nostrand, 1963.

WHITE, S. H. Evidence for a hierarchical arrangement of learning processes. In L. P. Lipsitt and C. C. Spiker (Eds.), Advances in child development and behavior. Vol. 2. New York: Academic Press, 1965. NOTE

1. The research reported herein was Project S-061, supported by the U.S. Department of Health, Education, and Welfare. 